\title{
PHENOMENOLOGY OF GEOMETRICAL FLAVOUR INTERACTIONS AT TeV ENERGIES
}

\author{
A. RINGWALD*, F. SCHREMPP and C. WETTERICH \\ Deutsches Elektronen Synchrotron-DESY, Hamburg, Germany \\ Received 22 October 1990 \\ (Revised 17 June 1991)
}

\begin{abstract}
We investigate the experimental signatures of the recently proposed "geometrical" production of many $\mathrm{W}, \mathrm{Z}$, Higgs and (primordial) fermions $\left(n_{\mathrm{w}} \approx \alpha_{\mathrm{w}}^{-1} \approx 30\right)$ with a relatively large cross section. We believe that such events, if they exist, stand a very good chance to be seen at the LHC (SSC) provided that the (parton) threshold energy for the onset of geometrical flavour production is below $11(28) \mathrm{TeV}$.
\end{abstract}

\section{Introduction}

The similar structure of infrared divergences in QCD and QFD has led [1] to the speculation that at very high energies weak and strong interactions should behave similarly. This analogy refers not only to the production of a few particles with large transverse momenta which is well described by (parton model) perturbation theory, but also to the large, essentially constant total inelastic cross section where perturbation theory presumably fails. The central argument of ref. [1] establishes that the total weak inelastic cross section as well as suitable total multiparticle cross sections (for producing a number of weakly interacting particles greater than a certain minimal number $n_{0}$ ) become approximately energy-independent in the asymptotic regime at very high energies,

$$
\sigma_{\mathrm{w}}=4 \pi c_{\mathrm{w}} m_{\mathrm{w}}^{-2} .
$$

(The quantity $c_{w}$ typically involves logarithms or small powers of energy.)

The magnitude $\left(c_{w}\right)$ of the asymptotic cross section as well as the mean multiplicity in asymptotic weak inelastic scattering processes are, however, not directly determined by arguments based on the structure of infrared divergences. By analogy with QCD it was speculated that

(i) Typical multiparticle cross sections are in the range $0.1 \mathrm{nb}-10 \mu^{\mathrm{r}}$ tor asymptotic parton-parton c.m. energies $\sqrt{\hat{s}}$.

*Address after October 1st, 1990: CERN, Geneva, Switzerland. 
(ii) The mean multiplicity is high, typically of order $1 / \alpha_{w} \simeq 30^{\star}$.

(iii) The inclusive differential cross sections decrease rapidly for transverse momenta exceeding a characteristic value of order $m_{\mathrm{w}}$.

(iv) The asymptotic behaviour sets in rather rapidly above a certain threshold energy of the order of a few $\mathrm{TeV}$ to about $20 \mathrm{TeV}$.

In a simple picture these processes arise from the scattering of the clouds of weak gauge bosons and scalars carried along by colliding quarks or leptons. Both the almost constant multiparticle cross sections and the characteristic $p_{t}$ distribution directly reflect the finite size of these clouds $\left(\alpha m_{\mathrm{w}}^{-1}\right)$ which is, of course, related to the finite range of the underlying interactions. We therefore call processes with these characteristics Geometric Flavour Interactions (GFI). In collisions with low $p_{\mathrm{t}}<m_{\mathrm{w}}$ the resolution is "bad", $\Delta x>m_{\mathrm{w}}^{-1}$. Therefore, only the gross geometrical features are relevant here, namely, the "weak size" of quarks or leptons corresponding to the size of the W, Z, Higgs, clouds and the "opacity" of the colliding objects which is measured by the factor $c_{\mathrm{w}}$ in eq. (1.1). Within such a geometrical scattering scenario our most speculative assumptions concern the high multiplicity and the large value of $c_{\mathrm{w}}$. (The "opaqueness" of the colliding objects is actually related to the large multiplicity.) Intuitively, one may envisage that a non-perturbative behaviour of the multiparticle cross sections arises from the strong "flavourelectric" and "flavourmagnetic" fields created by the presence of more than $\alpha_{w}^{-1}$ weak charges in a volume with a characteristic linear size $m_{w}^{-1}$. Much theoretical work will be needed to substantiate or falsify these speculations. In this paper we shall investigate the experimental possibilities to test this scenario. We leave aside here the theoretically more conservative but nevertheless experimentally interesting alternative that the asymptotically almost constant total inelastic cross section is dominated by the perturbative production of only a few weakly interacting particles.

In a different approach it was argued [3-6] that baryon $(B)$ and lepton $(L)$ number violating processes may become large at energies exceeding the sphaleron [7] energy $\left(M_{\mathrm{sp}} \simeq 10 \mathrm{TeV}\right)$. In particular, McLerran et al. [6] proposed that the strongly rising $(B+L)$-violating cross sections obtained from the instanton [8-10] calculation $[4-6,11-13]$

$$
\begin{gathered}
\sigma_{\Delta(B+L)} \sim \exp \left(-\frac{4 \pi}{\alpha_{\mathrm{w}}}\right) \exp \left[\frac{\sqrt{\hat{s}}}{M_{0}}\right]^{4 / 3}, \\
M_{0}=\frac{2}{3}\left(\frac{2 \pi}{\alpha_{\mathrm{w}}}\right)^{1 / 4} m_{\mathrm{w}}=195 \mathrm{GeV}
\end{gathered}
$$

\footnotetext{
^ Perturbation theory also indicates almost constant asymptotic cross sections [1,2], but is presumably inadequate to describe processes with large multiplicity $\sim \alpha_{w}^{-1}$.
} 
are qualitatively valid until $\sqrt{\hat{s}}$ reaches the s-wave unitarity bound at $\sqrt{\hat{s}} \simeq M_{1}$,

$$
M_{1} \simeq 16 \mathrm{TeV}
$$

They argued that for $\sqrt{\hat{s}}$ exceeding $M_{1}$ the strong s-wave amplitude leading to (1.2) proliferates to other partial waves and $(B+L)$-conserving channels (as a result of multi-instanton configurations) thereby unitarizing the amplitudes and inducing large forward peaked cross sections in the asymptotic regime $\sqrt{\hat{s}} \gg M_{1}$. This scenario predicts a sharp threshold for the onset of strong flavour interactions at $\sqrt{\hat{s}} \simeq M_{1}$ as well as a high mean multiplicity of weakly interacting particles,

$$
\begin{aligned}
& \bar{n}_{\mathrm{w}}=\frac{1}{3}\left(\frac{\sqrt{\hat{s}}}{M_{0}}\right)^{4 / 3} \text { for } \sqrt{\hat{s}} \leqslant M_{1} \\
& \bar{n}_{\mathrm{w}} \simeq 120 \text { for } \sqrt{\hat{s}} \simeq M_{1} .
\end{aligned}
$$

The typical asymptotic cross section was conjectured [6] to behave as

$$
\sigma_{\Delta(B+L)} \sim \frac{4 \pi}{M_{\mathrm{sp}}^{2}} \ln ^{\delta}\left(\frac{\sqrt{\hat{s}}}{M_{\mathrm{sp}}}\right)=50 \mathrm{pb}
$$

A distinctive feature of this scenario is a strong cross section for high multiplicity production of weakly interacting particles even at large angles in the threshold region $\sqrt{\hat{s}} \simeq M_{1}$, where the s-wave contribution should be substantial.

Unfortunately, the instanton calculation becomes unreliable at energies near $M_{1}$ and there are at least two other alternative scenarios consistent with $(B+L)$ violation at low energies (1.2) and asymptotically constant weak cross .sections (1.1):

(a) The $(B+L)$-violating cross sections remain always suppressed compared to the $(B+L)$-conserving cross sections. For a very weak $(B+L)$-violation the geometrical flavour interactions (1.1) would not be related to $(B+L)$-violation or instanton configurations.

(b) The instanton computation [4-6,11-13] for the cross section (1.2) breaks down well below the unitarity limit, but $(B+L)$-violating effects become nevertheless strong for high-energy scattering in the forward direction. We would then expect a similar phenomenology as for the geometrical flavour production [1], with the addition that a substantial fraction of the events violate $B+L$. The discussion in this paper will cover this case as well as the possible large angle (s-wave) multiparticle production arising in the scenario of ref. [6].

In any case, we should emphasize that a connection between the constant asymptotic cross section (1.1) and instantons [14] (or similar modified euclidean 
field configurations) would imply both a high mean multiplicity and a sharp threshold for the onset of geometrical flavour interactions.

Despite the considerable theoretical uncertainty of these speculations we think it is worthwhile to work out their experimental consequences. This is the aim of this paper. We will take (i)-(iv) as our working hypotheses. In sect. 2 we attempt a rough estimate for the typical multiplicity of weakly interacting particles at $\mathrm{TeV}$ energies and for the threshold energy necessary for the onset of geometrical flavour production. As a tool, we employ an analogy to strong interactions of hadrons. Due to the non-perturbative nature of the investigated phenomena, these "estimates" are at best "educated guesses". They set, however, the stage for the subsequent phenomenological discussion. In sect. 3 we estimate event rates for geometrical flavour production in proton-proton collisions, while sect. 4 is devoted to "bulk" characteristics like the total deposited transverse energy and average values of transverse and longitudinal momenta. In sect. 5 we add the additional, plausible working hypothesis of a flat rapidity distribution for GFI events. From this we derive the main characteristics of the event topology. We then estimate in sect. 6 the number of isolated electrons and muons per event and evaluate in sect. 7 the total missing transverse energy due to the escape of neutrinos. The jet activity both in the central and forward regions is discussed in sect. 8 . We summarize our results on the characteristic features of the signal by proposing appropriate experimental triggers in sect. 9 and briefly comment in sect. 10 on the exciting possibility that the LHC and SSC may become W, Higgs and top factories. A short discussion on possible signals for $B+L$ violation follows in sect. 11. The conclusions are presented in sect. 12 .

\section{Critical energy and multiplicity}

Weakly interacting particles produced by geometrical flavour interactions at high energies comprise $\mathrm{W}$ and $\mathrm{Z}$ bosons, photons, Higgs bosons, leptons, and quarks. Quarks will appear as jets and we count jets as particles. The fundamental coupling of all of these particles is governed by the weak gauge coupling $g_{w}$. One may guess that in a first approximation all particle species are produced in similar abundances. Alternatively, it is conceivable that the mechanism of geometrical flavour interactions mainly involves gauge bosons and Higgs scalars. In this case the production rate for "primordial" quarks and leptons (from off-shelf gauge bosons) is possibly suppressed by Fermi statistics for fermions of the same species or even by an additional factor $\alpha_{w}$ for each primordial fermion pair ${ }^{\star}$.

The phenomenon of multiparticle production with a large, constant or even somewhat increasing cross section $\left(\sigma_{\mathrm{w}} \sim A+C \ln ^{2} \hat{s}\right.$ or $\left.\sigma_{\mathrm{w}} \sim \hat{s}^{\hat{\alpha} / 2}, 0<\hat{\alpha} \ll 1\right)$ is presumably related to the breakdown of perturbation theory for large particle

\footnotetext{
${ }^{\star}$ No such suppression is expected for the final state fermions in $(B+L)$-violating processes.
} 
numbers, namely when $n_{\mathrm{w}} \alpha_{\mathrm{w}}$ becomes large. We explore the notion that GFI events set in rather abruptly once the number of produced particles exceeds a certain critical number, $n_{\mathrm{w}}>n_{\mathrm{w}}^{\text {crit }}$ with

$$
n_{\mathrm{w}}^{\mathrm{crit}} \alpha_{\mathrm{w}}=\nu_{\mathrm{w}}
$$

and $\nu_{\mathrm{w}}$ some constant of order one. This requires in turn that the (parton) energy exceeds a critical (threshold) energy, $\sqrt{\hat{s}}>E_{\mathrm{w}}^{\text {crit. }}$. The threshold energy can be roughly guessed by requiring that $\sqrt{\hat{s}}$ must be large enough to produce $n_{\mathrm{w}}^{\text {crit }}$ particles with mass $m_{\mathrm{w}}$ without a strong phase space suppression. We therefore parametrize

$$
E_{\mathrm{w}}^{\mathrm{crit}}=f_{\mathrm{w}} n_{\mathrm{w}}^{\mathrm{crit}} m_{\mathrm{w}}=f_{\mathrm{w}} \nu_{\mathrm{w}} m_{\mathrm{w}} / \alpha_{\mathrm{w}}
$$

The critical energy and the critical multiplicity are proportional to each other. The proportionality constant $f_{\mathrm{w}}$ corresponds to the mean energy per particle in units of its mass at the threshold $\star$. We will see later (sect. 4) that $f_{\mathrm{w}}$ plays an important rôle in the phenomenology of these processes.

Besides $f_{\mathrm{w}}$, the quantity $\nu_{\mathrm{w}}$ in (2.1) remains in this simple scenario the main unknown which can not be determined at present. It is a product of charge times coupling constant (as $\mathrm{Z} \alpha$ in $\mathrm{QED}^{\star \star}$ ) and can be interpreted as an indirect measure for a critical field strength responsible for the onset of non-perturbative behaviour.

It may be interesting to draw an analogy to QCD above and around the threshold $E_{\mathrm{s}}^{\text {crit }}$ where the asymptotic behaviour of cross sections sets in rather suddenly. For exotic s-channels without resonances $\left(\mathrm{K}^{+} \mathrm{N}, \mathrm{pN}, \ldots\right)$ one typically finds $E_{\mathrm{s}}^{\text {crit }} \simeq(1.9-2.2) \mathrm{GeV}$. Typical hadronic multiplicities are low near the threshold, $n_{\mathrm{s}}^{\text {crit }} \simeq 3^{\star \star \star}$, and we define again

$$
n_{\mathrm{s}}^{\text {crit }} \alpha_{\mathrm{s}}=\nu_{\mathrm{s}} .
$$

* We did not pay attention here to the fact that most fermion masses are much smaller than $m_{\mathrm{w}}$. Light fermion masses have the tendency to lower $E_{w}^{\text {crit }}$. One may account for different particle masses by replacing $m_{\mathrm{w}}$ in (2.2) by a suitable average mass $\bar{m}$.

$\star \star T h e r e$ is some similarity between $\nu_{\mathrm{w}}$ and the quantity $Z \alpha$ relevant for the breakdown of perturbative QED in the scattering of highly charged ions. A large number of final state particles comes together with strong flavour fields. In contrast to heavy ion scattering, however, the large particle numbers occur here only in the final state. An important difference for multiparticle production in QED and QFD arises from the fact that the photon clouds carry no electric charge and do not interact (except for loop effects). All electrically charged particles below the $\mathrm{W}$ boson threshold are fermions. Multi-electron production in QED is therefore hindered by the large Fermi energy required to concentrate $\alpha^{-1}$ electrons in a small volume.

$\star \star \star$ We derive this number from an extrapolation of average hadron multiplicities [15] down to the threshold energy, rather than from the trivial fact that at least three final state particles must occur for inelastic scattering in exotic channels. 
Let us speculate that we can use in (2.1) the same value as for QCD, i.e. $\nu_{w}=\nu_{\mathrm{s}}$. We then infer from $\alpha_{s}(2 \mathrm{GeV}) \simeq 0.31-0.37$ and $\alpha_{\mathrm{w}}(5 \mathrm{TeV}) \simeq 1 / 31$

$$
\begin{aligned}
\nu_{\mathrm{s}} & \simeq 1, \\
n_{\mathrm{w}}^{\text {crit }} & \simeq 30 .
\end{aligned}
$$

Eq. (2.2) has an analogy for strong hadronic interactions,

$$
f_{\mathrm{s}} n_{\mathrm{s}}^{\text {crit }} \Lambda_{\mathrm{s}}=E_{\mathrm{s}}^{\text {crit }} \simeq 2 \mathrm{GeV},
$$

with $\Lambda_{\mathrm{s}}$ some typical QCD scale corresponding, for example, to the average mass of the produced hadrons. In $\mathrm{QCD}, f_{\mathrm{s}}$ represents essentially a phase space factor since enough kinetic energy per particle is needed to avoid threshold suppression. We determine $f_{\mathrm{s}}$ phenomenologically from the average kinetic energy/mass near the threshold,

$$
1 \leqslant f_{\mathrm{s}}<1.5 \text {. }
$$

Assuming $f_{\mathrm{w}} \simeq f_{\mathrm{s}}$ and $\nu_{\mathrm{w}} \simeq \nu_{\mathrm{s}}$ one estimates from (2.2)

$$
E_{\mathrm{w}}^{\mathrm{crit}} \simeq(2.5-4) \mathrm{TeV} \text {. }
$$

For $\sqrt{\hat{s}}$ larger than the threshold energy $E_{\mathrm{w}}^{\text {crit }}$ we only expect a slow increase of the mean multiplicity $\bar{n}$ (with logarithms or small powers of $\hat{s}$ ). One may even speculate that the crucial parameter for non-perturbative forward scattering at very high energies is $\alpha \bar{n}$ and infer a general scaling relation between mean (parton) multiplicities ${ }^{\star}$ in strong hadronic interactions $\bar{n}_{\mathrm{s}}$ and for the corresponding quantity in geometrical flavour interactions $\bar{n}_{\mathrm{w}}$,

$$
\left.\left.\alpha_{\mathrm{w}} \bar{n}_{\mathrm{w}}\right|_{\sqrt{\S}} \propto \alpha_{\mathrm{s}} \bar{n}_{\mathrm{s}}\right|_{\sqrt{\S} \Lambda_{\mathrm{s}} / m_{\mathrm{w}}} .
$$

For our phenomenological analysis we shall concentrate on values of $E_{\mathrm{w}}^{\text {crit }}$ and $n_{\mathrm{w}}^{\text {crit }}$ in the range defined by the estimates (2.8), (2.5) and (1.4), (1.5). Bearing in mind all the uncertainties we shall explore here the possibility that typical GFI events contain at least 30 weakly interacting particles ${ }^{\star \star \star}$. We shall, actually, use $n_{\mathrm{w}}=30$

* The use of hadron multiplicities for an analogy between $\nu_{w}$ and $\nu_{s}$ is problematic. The most straightforward analogous quantity would be the multiplicity of partons with transverse momenta below $1 \mathrm{GeV}$ in pp scattering near threshold. Unfortunately, partons are not well defined in the non-perturbative regime. In any case, there appears no direct indication that $\nu_{\mathrm{s}}$ is substantially bigger than one (e.g. $2 \pi$, [14]), since this would suggest a higher threshold with a production of many hadrons at the threshold.

$\star \star$ This analogy concerns low-p partons in quark-(anti)quark scattering rather than high-p jet production in $\mathrm{e}^{+} \mathrm{e}^{-}$annihilation etc., which is well described by perturbation theory.

$\star \star \star$ As an alternative scenario, one could also imagine that the transition from perturbative QFD to non-perturbative many particle production is much smoother than in QCD. In this case the multiplicity may increase more steadily over a larger range in $\sqrt{\hat{s}}$, starting from the production of only a few weakly interacting particles at low $\sqrt{\hat{s}}$. 
for the remainder of this paper with all results rescaled easily for the experimentally even more advantageous case of higher multiplicity $n_{\mathrm{w}}>30$.

\section{Cross sections and production rates}

For a discussion of geometrical flavour production at future proton-proton colliders we start from the working hypothesis that the parton cross section $\sigma_{w}(\hat{s})$ becomes almost constant above the threshold $E_{\mathrm{w}}^{\mathrm{crit}}$, with a size of order

$$
\sigma_{\mathrm{w}}^{0}=0.1 \mathrm{nb}-10 \mu \mathrm{b} \text {, }
$$

as estimated in ref. [1]. In particular, let us assume in this paper a relatively sudden onset of geometrical QFD cross sections for $\sqrt{\hat{s}} \geqslant E_{\mathrm{w}}^{\text {crit }}$ and approximate

$$
\sigma_{\mathrm{w}}(\sqrt{\hat{s}})=\sigma_{\mathrm{w}}^{0} \theta\left(\sqrt{\hat{s}}-E_{\mathrm{w}}^{\mathrm{crit}}\right)
$$

It is then easy to estimate the event rate for the production of many weakly interacting particles in proton-proton collisions

$$
\begin{gathered}
N / \Delta t=\mathscr{L} \sum_{i j} \sigma_{i j}^{0} \hat{\mathscr{L}}_{i j}\left(E_{\mathrm{w}}^{\mathrm{crit}} / \sqrt{s}\right), \\
\hat{\mathscr{L}}_{i j}\left(\sqrt{\tau_{\text {crit }}}\right)=\int_{\tau_{\text {crit }}}^{1} \mathrm{~d} \tau \frac{\mathrm{d} \hat{\mathscr{L}}_{i j}}{\mathrm{~d} \tau} \\
\frac{\mathrm{d} \hat{\mathscr{L}}_{i j}}{\mathrm{~d} \tau}=\frac{1}{1+\delta_{i j}} \int_{\tau}^{1} \frac{\mathrm{d} x}{x}\left(f_{i}(x) f_{j}(\tau / x)+f_{i}(\tau / x) f_{j}(x)\right) .
\end{gathered}
$$

Here $i, j$ refer to the weakly interacting parton species which include (anti) quarks but no gluons. The corresponding structure functions $f_{i}(x)$ are evaluated at $Q^{2} \simeq m_{\mathrm{w}}^{2}$ and $\tau=\hat{s} / \mathrm{s}, \tau_{\text {crit }}=\left(E_{\mathrm{w}}^{\text {crit }}\right)^{2} / \mathrm{s}$. With a luminosity $\mathscr{L}=10^{33} \mathrm{~cm}^{-2} \mathrm{~s}^{-1}$ and $\sigma_{i j}^{0} \simeq 1 \mathrm{nb}$ independent of the parton type one finds that $\hat{\mathscr{L}}=\sum_{i j} \hat{\mathscr{L}}_{i j}$ gives the number of events per second. In this case the limit of detectability should be around $\hat{\mathscr{L}}=10^{-5}$ which corresponds to 100 events per year. We have computed $\hat{\mathscr{L}}$ as a function of the threshold energy $E_{w}^{\text {crit }}$ for LHC and SSC energies and for $\sqrt{s}=200 \mathrm{TeV}$ (ELOISATRON) (fig. 1). For small $E_{\mathrm{w}}^{\text {crit }} / \sqrt{s}$ the quark-antiquark sea gives the dominant contribution. We conclude that GFI events can be seen for $E_{\mathrm{w}}^{\text {crit }}<11(28) \mathrm{TeV}$ for LHC (SSC) if $\sigma_{\mathrm{w}}^{0}$ is around $1 \mathrm{nb}$. Thus the SSC should be able to detect or reject this effect if the signal can be clearly separated from the QCD background. The possibility for detection at the LHC depends crucially on the precise values of $E_{\mathrm{w}}^{\mathrm{crit}}$ and $\sigma_{\mathrm{w}}^{0}$. For $\sigma_{\mathrm{w}}^{0}=100$ (1) $\mathrm{pb}$ the window for the threshold energy shrinks to $E_{w}^{\text {crit }}<10$ (7) TeV. A future collider in the $100 \mathrm{TeV}$ 


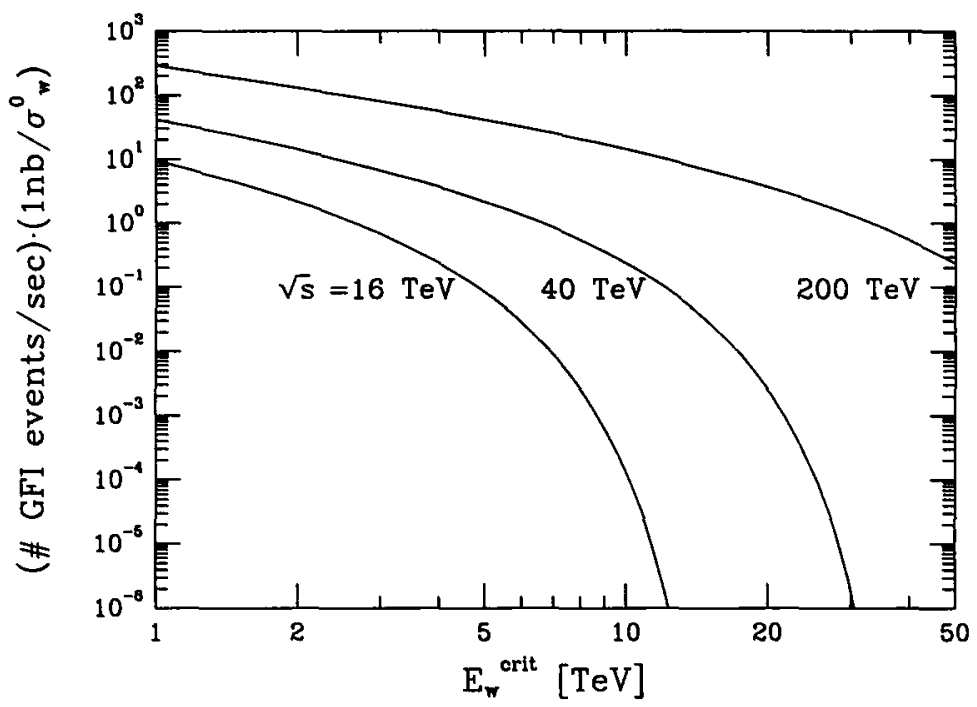

Fig. 1. Number of GFI events per second as a function of the (parton) threshold energy $E_{\mathrm{w}}^{\text {crit }}$. We assume a constant cross section $\sigma_{\mathrm{w}}^{0}=1 \mathrm{nb}$ for weakly interacting partons with energies above $E_{\mathrm{w}}^{\text {crit }}$ and a pp luminosity of $\mathscr{L}=10^{33} \mathrm{~cm}^{-2} \mathrm{~s}^{-1}$.

range (ELOISATRON) would be needed for a full exploration of the asymptotic behaviour beyond the threshold energy.

\section{Transverse momentum and energy}

At very high parton energies the characteristic transverse momentum of the weakly interacting particles produced in GFI events presumably corresponds to the geometrical size $R_{\mathrm{w}}$ of the cloud of gauge bosons and scalars around the quarks. $R_{\mathrm{w}}$ is of the order of the inverse W boson mass. Let us exploit further the analogy to hadronic cross sections which are characterized by a universal exponential decrease in transverse mass $m_{\mathrm{t}}=\sqrt{m^{2}+p_{\mathrm{t}}^{2}}$. Correspondingly, we take the (inclusive) distribution

$$
\mathrm{d} \sigma_{\mathrm{w}} / \mathrm{d} p_{\mathrm{t}} \sim p_{\mathrm{t}} \exp \left(-R_{\mathrm{w}} \sqrt{m^{2}+p_{\mathrm{t}}^{2}}\right)
$$

for the transverse momentum of a "geometrically" produced particle with mass $m$. Remembering that for hadronic interactions $R_{\mathrm{s}} \simeq 2 \pi \sqrt{\alpha^{\prime}} \simeq 6 \mathrm{GeV}^{-1}$, (with universal Regge slope $\alpha^{\prime}=1 / 2 m_{\rho}^{2}$ ), we expect

$$
R_{\mathrm{w}} \simeq \sqrt{2} \pi / m_{\mathrm{w}}
$$


This gives an average transverse momentum for a produced $\mathrm{W}$

$$
\bar{p}_{\mathrm{t}} \simeq 2^{1 / 4} m_{\mathrm{w}} / 2 \simeq 48 \mathrm{GeV} .
$$

The average energy (or longitudinal momentum ${ }^{\star}$ ) in the p-p c.m.s. per particle produced in GFI events is inversely proportional to the multiplicity $\star \star$

$$
\langle E\rangle=\left\langle\frac{x}{\bar{n}_{\mathrm{w}}(\sqrt{\hat{s}})}\right\rangle \sqrt{s} \simeq \frac{\sqrt{s}}{n_{\mathrm{w}}}\langle x\rangle \simeq\left\langle p_{\mathrm{L}}\right\rangle
$$

with

$$
\langle x\rangle=\frac{1}{2}\left\langle x_{\mathrm{a}}+x_{\mathrm{b}}\right\rangle=\frac{\sum_{i j} \int \mathrm{d} x_{\mathrm{a}} \mathrm{d} x_{\mathrm{b}} \theta\left(x_{\mathrm{a}} x_{\mathrm{b}}-\left(E_{\mathrm{w}}^{\text {crit }}\right)^{2} / s\right) f_{i}\left(x_{\mathrm{a}}\right) f_{j}\left(x_{\mathrm{b}}\right)\left[\left(x_{\mathrm{a}}+x_{\mathrm{b}}\right) / 2\right]}{\sum_{i j} \int \mathrm{d} x_{\mathrm{a}} \mathrm{d} x_{\mathrm{b}} \theta\left(x_{\mathrm{a}} x_{\mathrm{b}}-\left(E_{\mathrm{w}}^{\text {crit }}\right)^{2} / s\right) f_{i}\left(x_{\mathrm{a}}\right) f_{j}\left(x_{\mathrm{b}}\right)} .
$$

Here the sum again extends over weakly interacting partons (no gluons). Let us consider four typical values of $\sqrt{s}$ and $E_{\mathrm{w}}^{\text {crit }}$
(a) $\mathrm{LHC}(1)$ :
$\sqrt{s}=16 \mathrm{TeV}$,
$E_{\mathrm{w}}^{\mathrm{crit}}=3.5 \mathrm{TeV}$,
(b) $\mathrm{LHC}(2)$ :
$\sqrt{s}=16 \mathrm{TeV}$,
$E_{\mathrm{w}}^{\text {crit }}=10 \mathrm{TeV}$,
(c)
$\sqrt{s}=40 \mathrm{TeV}$,
$E_{\mathrm{w}}^{\text {crit }}=10 \mathrm{TeV}$,
(d) ELO:
$\sqrt{s}=200 \mathrm{TeV}$
$E_{\mathrm{w}}^{\text {crit }}=10 \mathrm{TeV}$,

for which we find (cf. fig. 2)

$$
\begin{aligned}
& \langle x\rangle \simeq 0.30,0.67,0.33 \text { and } 0.13, \\
& \left.\langle E\rangle \simeq 160,360,440 \text { and } 870 \mathrm{GeV} \text { (for } n_{\mathrm{w}}=30\right),
\end{aligned}
$$

respectively.

Bulk quantities like $\langle E\rangle$ in (4.4) give, however, only very rough information on the distribution of scattering angles. For example, the average values $\left\langle p_{t}\right\rangle \simeq 48$ $\mathrm{GeV},\langle E\rangle \simeq 870 \mathrm{GeV}$ for a $200 \mathrm{TeV}$ machine are perfectly consistent with a substantial fraction of events scattered at angles much wider than corresponding to $\left\langle p_{\mathrm{t}}\right\rangle\left\langle\left\langle p_{\mathrm{L}}\right\rangle\right.$. On the other hand, small values of $\langle E\rangle$ for LHC or SSC together

* The approximation of the longitudinal momentum $p_{L}=\sqrt{E^{2}-p_{t}^{2}-m^{2}}$ by the energy is of course only valid for $p_{\mathrm{L}}^{2} \gg p_{\mathrm{l}}^{2}+m^{2}$ corresponding to $\tan \vartheta=\sqrt{p_{\mathrm{l}} / p_{\mathrm{L}}}<0.2$ for $\mathrm{W}$ bosons with $p_{1}<50$ $\mathrm{GeV}$.

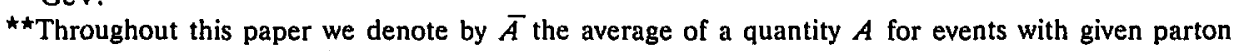
kinematics, whereas $\langle A\rangle$ denotes averages in pp scattering with the (anti) quark distributions in the proton folded in. In particular one has $\left\langle p_{l}\right\rangle=\bar{p}_{1}$. 


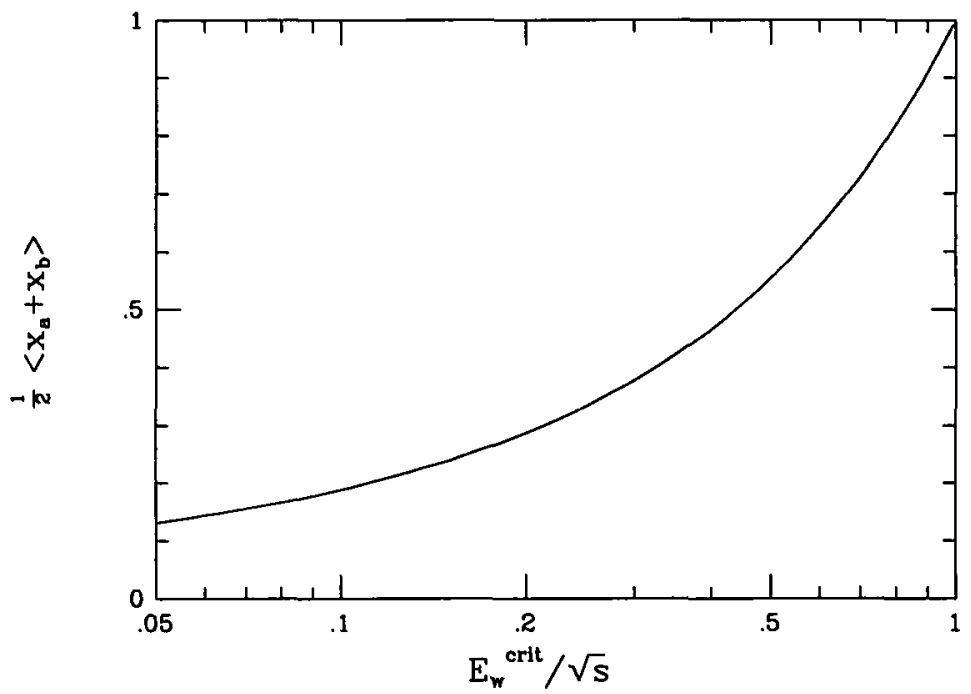

Fig. 2. Average total parton energy in the p-p c.m.s., $\frac{1}{2}\left\langle x_{\mathrm{a}}+x_{\mathrm{b}}\right\rangle=\left\langle E_{\mathrm{parl}}\right\rangle / \sqrt{s}$, plotted as a function of the parton threshold energy $E_{\mathrm{w}}^{\text {crit }} / \sqrt{s}$, both quantities in units of $\sqrt{s}$.

with transverse momenta of a few tens of $\mathrm{GeV}$ certainly imply that many events involve relatively "large" angle scattering.

Consider events with given parton kinematics $\left(x_{\mathrm{a}}, x_{\mathrm{b}}\right)$. For a fixed $p_{\mathrm{t}}$ large scattering angles arise from small longitudinal momenta. In turn, $p_{\mathrm{L}}$ is bounded by the fact that the total available parton energy has to be distributed among $n_{\mathrm{w}}$ particles. If $\bar{n}_{\mathrm{w}}$ varies only slowly for $\sqrt{\hat{s}}>E_{\mathrm{w}}^{\text {crit }}$, comparatively large angles arise from partons which have a total energy just above $E_{w}^{\text {crit }}$. Such partons are present in $\mathrm{p}-\mathrm{p}$ collisions at arbitrary $\sqrt{s}$ (provided the energy is sufficient to have enough partons with $\sqrt{\hat{s}}>E_{\mathrm{w}}^{\text {crit }}$ ). The corresponding kinematical region is characterized by a total parton energy in the c.m.s. of the p-p collisions, $E=\frac{1}{2}\left(x_{\mathrm{a}}+x_{\mathrm{b}}\right) \sqrt{s}$, near $E_{\mathrm{w}}^{\text {crit }}$ and $\sqrt{\hat{s}} \geqslant E_{\mathrm{w}}^{\text {crit }}$, i.e. $x_{\mathrm{a}} \simeq x_{\mathrm{b}}$. The total available kinetic energy for partons with kinematics near the threshold is directly related to the average energy $E$ per average mass $m$ of the produced particles,

$$
f_{\mathrm{w}}=\frac{E_{\mathrm{w}}^{\mathrm{crit}}}{n_{\mathrm{w}}^{\text {crit }} \bar{m}} \simeq \frac{\bar{E}}{\bar{m}} .
$$

(Here we have replaced $m_{\mathrm{w}}$ in (2.2) by a suitable average over the masses of all produced particles.) Values of $f_{\mathrm{w}}$ near one are synonymous with the occurence of large angle scattering in geometrical flavour production. Only for high values of $f_{\mathrm{w}}$ or for collisions not dominated by threshold events are more detailed assumptions about the rapidity distribution (see sect. 5) needed in order to assert whether the 
events look central or forward/backward peaked.

The $\mathrm{W}, \mathrm{Z}$ bosons, Higgs scalars and top quarks will not be directly observable in the detectors of future pp colliders. They decay into jets and leptons. Let $l_{\mathrm{w}}$ denote the number of light quarks and leptons produced in GFI events, i.e. those particles with mass much smaller than $\left\langle p_{\mathrm{t}}\right\rangle$, including the bottom quark. We then expect

$$
l_{\mathrm{w}} / n_{\mathrm{w}} \simeq 1.5-2,
$$

depending somewhat on the ratio of primordial light fermions to light fermions originating from

(i) W, Z, and Higgs scalar decay (2 light fermions /W, Z or scalar (if $m_{\mathrm{h}}<2 m_{\mathrm{w}}$ ));

(ii) top decay (3 light fermions/top).

The calorimeters at future $\mathrm{TeV}$ colliders will measure directly the energy and angular distributions of these light fermions ${ }^{\star}$.

We concentrate on events with fixed $l_{\mathrm{w}}$ (and fixed parton kinematics $x_{\mathrm{a}}, x_{\mathrm{b}}$ ). Due to the decay of the heavy particles the average transverse momentum per light fermion will be somewhat smaller than (4.3). We take this into account by using (4.1) with $m=0$, which results in

$$
\bar{p}_{\mathrm{t}}^{1}=2 / R_{\mathrm{w}} \simeq 36 \mathrm{GeV} .
$$

We estimate the average total transverse energy per GFI event for fixed $l_{\mathrm{w}}$ (between 45 and 60 ) assuming uncorrelated $p_{\mathrm{t}}$ distributions of the final state particles,

$$
\bar{E}_{\mathrm{t}}^{\text {tot }}=l_{\mathrm{w}} \bar{p}_{\mathrm{t}}^{\mathrm{l}} \simeq(1.6-2.2) \mathrm{TeV} .
$$

It is much larger than for typical strong interaction events!

\section{Rapidity distribution and event topology}

Since at future $\mathrm{TeV}$ colliders triggering is far more advantageous in the central region, let us ask next what fraction of the "geometrically" produced flavoured particles we may expect there. As a typical central range of pseudo rapidities, $\eta=-\ln \tan \frac{1}{2} \vartheta$, we consider

$$
|\eta|<\eta_{0} \simeq 2.5
$$

corresponding to

$$
10^{\circ}<\vartheta<170^{\circ} .
$$

\footnotetext{
${ }^{\star}$ In the central angular region both $p_{\mathrm{L}}$ and $p_{\mathrm{t}}$ are of the order of $m_{\mathrm{w}}$ and the two jets from a W decay can probably be resolved. This becomes more difficult in the near-forward region where $p_{\mathrm{L}} \gg p_{1}, m_{\mathrm{w}}$.
} 
In case of "threshold" machines $\left(\sqrt{s}\right.$ near $\left.E_{\mathrm{w}}^{\text {cril }}\right)$ and small values of $f_{\mathrm{w}}<1.5$, the kinematical restrictions (4.7) on $p_{\mathrm{L}}$ directly lead us to expect many particles in this region for each event. Otherwise a more detailed estimate is necessary, requiring some plausible assumption on the rapidity distribution of the produced light fermions. For an ansatz about the rapidity distribution of an individual light fermion we again take recourse to the analogy with hadrons. Both a classical "multiperipheral" picture or data on multi-pion production suggest an essentially constant rapidity distribution in the parton c.m.s.

$$
\frac{\mathrm{d} \sigma}{\mathrm{d} \hat{y}}=\text { const. } \times \theta\left(y_{\max }-|\hat{y}|\right), \text { for } \sqrt{\hat{s}}>E_{\mathrm{w}}^{\text {crit }} .
$$

The next step is to estimate the rapidity interval $\left(y_{\max }\right)$ from our knowledge of the average energy per light particle in a given event

$$
\bar{E}=\frac{\sqrt{\hat{s}}}{l_{\mathrm{w}}}=\frac{\sqrt{s} \sqrt{x_{\mathrm{a}} x_{\mathrm{b}}}}{l_{\mathrm{w}}},
$$

which must coincide with

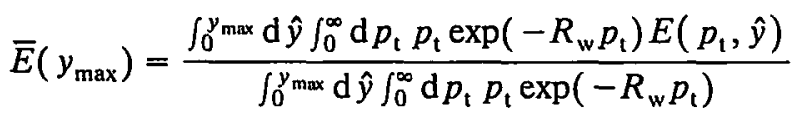

$$
\begin{aligned}
& =\bar{p}_{\mathrm{t}} ! \frac{\sinh y_{\max }}{y_{\max }},
\end{aligned}
$$

where, for $m^{2} \ll p_{1}^{2}$,

$$
E\left(p_{\mathrm{t}}, \hat{y}\right) \approx p_{\mathrm{t}} \cosh \hat{y} .
$$

We display $y_{\max }$ as a function of $\sqrt{\hat{s}} / \bar{E}_{\mathrm{t}}^{\text {tot }}=\sqrt{x_{\mathrm{a}} x_{\mathrm{b}} s} /\left(l_{\mathrm{w}} \bar{p}_{\mathrm{t}}^{\prime}\right)$ in fig. 3. For fixed $\bar{E}_{\mathrm{t}}^{\text {tot }}=2 \mathrm{TeV}$ the maximum length of the rapidity interval (for $\hat{s}=s$ ) is given by $y_{\max }=4.2,5.4$ and 7.3 for the LHC, SSC and ELOISATRON, respectively, whereas one finds $y_{\max }=1.9(3.6)$ for $\sqrt{\hat{s}}=E_{\mathrm{w}}^{\text {crit }}=3.5$ (10) TeV.

The probability $z$ of finding a given light particle (a muon, say) in the central detector range (5.1), can now be determined by shifting the rapidity interval from the parton c.m.s. to the $p-p$ c.m.s. according to

$$
\delta y=\tanh ^{-1} \beta, \text { with } \beta=\frac{x_{\mathrm{a}}-x_{\mathrm{b}}}{x_{\mathrm{a}}+x_{\mathrm{b}}},
$$




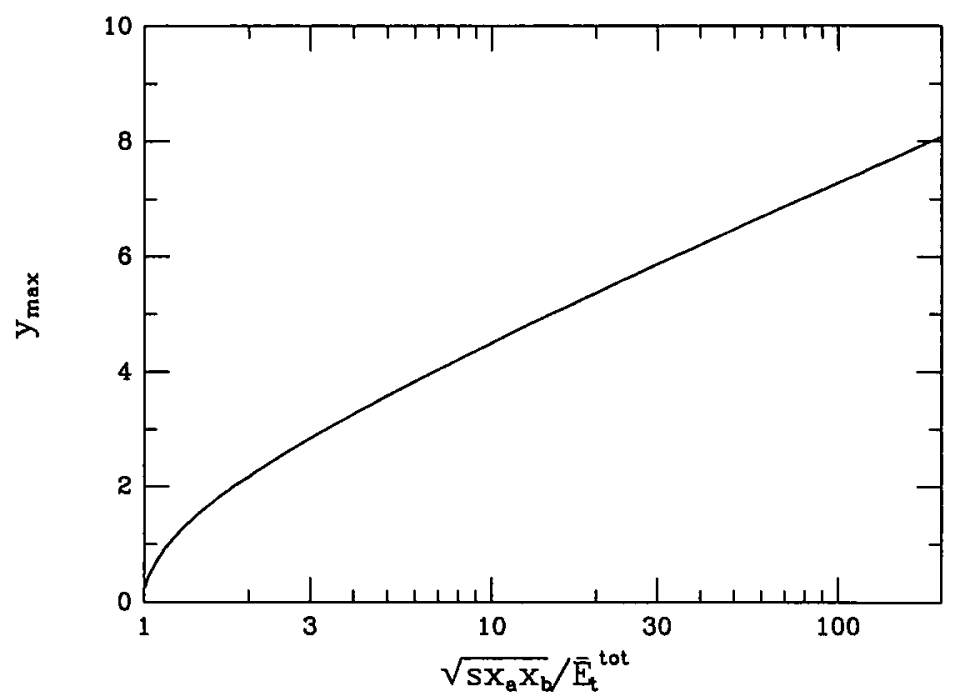

Fig. 3. Size of the rapidity interval in the parton c.m.s. as a function of the total parton c.m. energy divided by the average total transverse energy of the produced light particles. We assume a constant rapidity distribution in the parton c.m.s. for $-y_{\max }<y<y_{\max }$.

and evaluating the overlap with the interval $\left[-\eta_{0}, \eta_{0}\right]$. One finds

$$
\begin{gathered}
z\left(x_{\mathrm{a}}, x_{\mathrm{b}}\right)=\frac{y_{\mathrm{l}} \theta\left(y_{\mathrm{l}}\right)}{y_{\max }}, \\
y_{1}=\frac{1}{2}\left[\min \left(\eta_{0}, y_{\max }+\delta y\right)+\min \left(\eta_{0}, y_{\max }-\delta y\right)\right] .
\end{gathered}
$$

Here we use eq. (5.7) and eqs. (5.4),(5.5) in order to evaluate $\delta y$ and $y_{\max }$ as a function of $x_{\mathrm{a}}, x_{\mathrm{b}}$. The mean value $\langle z\rangle$ is then obtained by averaging (5.8) with the parton distributions assuming that $l_{\mathrm{w}}$ can be taken constant to a good approximation.

If we assume, in addition, that for every given event the $l_{\mathrm{w}}$ light particles are equally distributed over the rapidity interval $\left[-y_{\max }, y_{\max }\right]$, then the number $l_{\text {centr. }}$ of particles in the "central" region (5.1) is given for every event by $z l_{w}$ and one obtains

$$
\left\langle l_{\text {centr. }} / l_{\mathrm{w}}\right\rangle=\langle z\rangle \text {. }
$$

We display $\left\langle l_{\text {centr. }} / l_{\mathrm{w}}\right\rangle$ in fig. 4 as a function of the dimensionless quantity $\rho=\sqrt{s} / \bar{E}_{\mathrm{t}}^{\text {tot }}$ for the four values of $E_{\mathrm{w}}^{\text {crit }} / \sqrt{s}$ specified in (4.6). For simple kinematical reasons, $\rho$ must be larger than $\sqrt{s} / E_{\mathrm{w}}^{\text {crit }}$. Apparently, even at $\sqrt{s}=200 \mathrm{TeV}$, there is a more than $50 \%$ chance to find a given muon (or other light particle) in the angular range (5.2)! In order to provide an impression about the angular distribution of the light fermions in a GFI event, we plot in fig. 5 the relative 


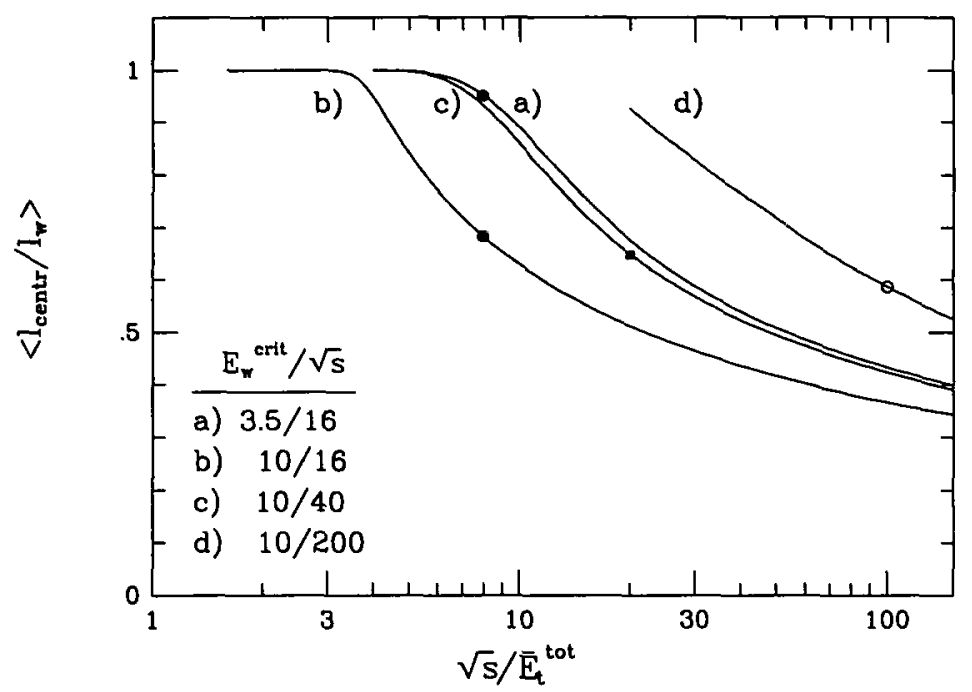

Fig. 4. Fraction of the number of light particles in the angular region, $10^{\circ}<\vartheta<170^{\circ}$, of a typical central detector, displayed versus $\sqrt{s} / \bar{E}_{\mathrm{t}}^{\text {tot }}$ for four representative values of $E_{\mathrm{w}}^{\text {crit }} / \sqrt{s}$. The values for a fixed number of light fermions, $l_{\mathrm{w}}=60$, are marked by solid circles $(\sqrt{s}=16 \mathrm{TeV})$, square $(\sqrt{s}=40$ $\mathrm{TeV})$ and open circle $(\sqrt{s}=200 \mathrm{TeV})$.

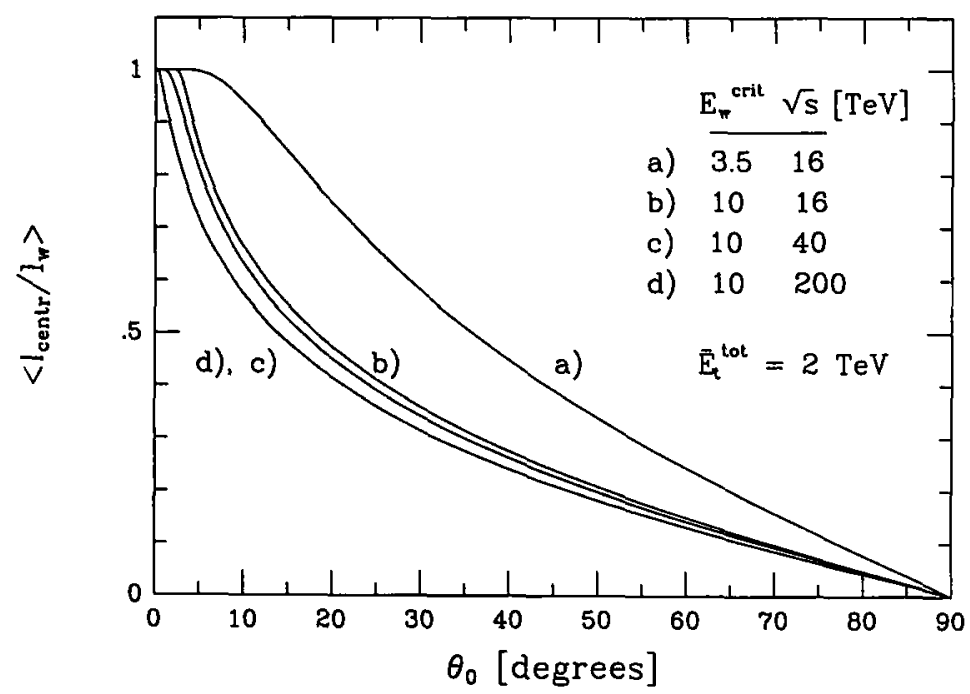

Fig. 5. Relative number of light particles in the angular region $\theta_{0}<\vartheta<180^{\circ}-\theta_{0}$ versus $\theta_{0}$ for fixed total transverse energy $\bar{E}_{\mathrm{l}}^{\text {tot }}=2 \mathrm{TeV}$ and the same values of $E_{\mathrm{w}}^{\text {crit }}$ and $\sqrt{\mathrm{s}}$ as in fig. 4 . 
number of particles in the angular region $\vartheta_{0}<\vartheta<180^{\circ}-\vartheta_{0}$, i.e. $\left\langle l_{\text {centr. }} / l_{\mathrm{w}}\right\rangle\left(\vartheta_{0}\right)$ $=\langle z\rangle\left(\vartheta_{0}\right)$, as a function of $\vartheta_{0}$ for a fixed value $\bar{E}_{\mathrm{t}}^{\text {tot }}=2 \mathrm{TeV}$. We find that the average "centrality" of the events, $z\left(\vartheta_{0}\right)$, depends strongly on the parton threshold energy $E_{\mathrm{w}}^{\text {crit. }}$. This effect arises since the average gets a large contribution from partons with "threshold kinematics" for which the "centrality" of the event essentially depends on $f_{\mathrm{w}}$ (cf. the discussion in sect. 4). Although our approximations (like fixed $l_{w}$, flat rapidity distribution, constant cross section above threshold) are very crude, we believe that quite generally, GFI events with large central activity will occur frequently as a result of the high sea quark densities at small $x$.

\section{Charged lepton multiplicity}

The cleanest signature of these events is probably the expected high number of isolated charged leptons in each event. Let us estimate the mean relative charged lepton multiplicity $R_{\mathrm{L}}$,

$$
R_{\mathrm{L}}=\left\langle\frac{\# \text { charged leptons }}{n_{\mathrm{w}}}\right\rangle
$$

Charged leptons arise here from three different sources: (i) primordial leptons; (ii) leptons from $\mathrm{W}$ and $\mathrm{Z}$ decays; (iii) leptons from Higgs decay. We neglect semileptonic top decays and do not count here the charged leptons from $b$ and $c$ quark decays within the quark jets. Our signature for leptons, therefore, consists of isolated leptons.

The average ratio of the number of electrons $\left(N_{\mathrm{e}}\right)$ or muons $\left(N_{\mu}\right)$ compared to the total number of light particles $l_{\mathrm{w}}$ can be estimated rather reliably. One has $N_{\mathrm{e}, \mu} / l_{\mathrm{w}}=1 / 20$ from $\mathrm{W}$ and $\mathrm{Z}$ decays, $N_{\mathrm{e}, \mu} / l_{\mathrm{w}} \simeq 0$ from Higgs decays, and $N_{\mathrm{e}, \mu} / l_{\mathrm{w}} \simeq 1 / 30$ from primordial fermion production. We estimate

$$
\left\langle\frac{N_{\mathrm{e}, \mu}}{l_{\mathrm{w}}}\right\rangle \simeq \frac{1}{20}-\frac{1}{25}
$$

giving an average of 2.4-3 electrons and 2.4-3 muons for $l_{\mathrm{w}}=60$. Photons with $p_{\mathrm{t}} \approx 30-40 \mathrm{GeV}$ are also expected and give an additional signal in the electromagnetic calorimeter.

We should emphasize that the electric charges of the leptons are essentially uncorrelated. This results in high probabilities to find several like-sign leptons. (For five charged leptons the probability of finding three, four or five like signs is around $10 / 16,5 / 16$, or $1 / 16$, respectively.) 


\section{Missing transverse momentum and energy}

The relative multiplicity of neutrinos equals the relative multiplicity of charged leptons. For $n_{\mathrm{w}}=30$ we thus expect around 5-9 neutrinos, each with an average transverse momentum of the order of $30-40 \mathrm{GeV}$. (Many neutrinos are decay products of $\mathrm{W}$ and $\mathrm{Z}$ bosons.) The neutrino transverse momenta partly cancel in the sum for the total missing momentum. Using (cf. eq. (6.2))

$$
\left\langle N_{\nu}\right\rangle \approx 3\left\langle N_{e, \mu} / l_{\mathrm{w}}\right\rangle l_{\mathrm{w}} \approx(0.12-0.15) l_{\mathrm{w}},
$$

we estimate (for $l_{\mathrm{w}}=60$ ) the total missing transverse momentum $\not p_{\mathrm{i}}^{\text {tot }}$ to be

$$
\left\langle\not p_{\mathrm{t}}^{\mathrm{tot}}\right\rangle \approx \sqrt{\left\langle N_{\nu}\right\rangle} \bar{p}_{\mathrm{t}}^{1} \approx 100 \mathrm{GeV} \text {. }
$$

The total missing energy can be inferred from (4.7), $Z^{\text {tot }} \approx\left\langle N_{\nu}\right\rangle\langle E\rangle$.

\section{Jet activity}

Most of the energy of a typical GFI event will appear in a multitude of jets. Indeed, the fraction of light quarks and $\tau$ jets in $l_{\mathrm{w}}$ can be estimated to be $N_{\mathrm{jet}} / l_{\mathrm{w}}=3 / 4,1$ and $5 / 6$ for gauge boson decay, scalar decay and primordial fermion production, respectively. Thus, we expect

$$
\left\langle\frac{\# \text { jets }}{l_{w}}\right\rangle \approx \frac{3}{4}-\frac{5}{6} .
$$

(We neglect here the possibility to resolve $\mathrm{b}$ and $\tau$ jets into individual jets corresponding to their decay products.) For $l_{\mathrm{w}}=60$, a typical event contains the enormous number of $45-50$ jets and even a conservative value $l_{\mathrm{w}}=40$ implies at least 30 jets! These numbers are so high that it becomes hard to resolve all the jets individually, especially, if much of the activity is in the forward direction.

Within the range of the central detector $(\eta<2.5)$, however, there should be a good chance to resolve almost all jets, given a typical jet resolution interval of $\sqrt{\Delta \phi^{2} \times \Delta \eta^{2}}=0.4$. The number of jets in this region can be found from fig. 4 , using \# central jets $=\#$ jets $\left\langle l_{\text {centr. }} / l_{\mathrm{w}}\right\rangle$. We see that more than 20 central jets are expected. Provided that they can be cleanly separated, there should be little background from strong interaction processes. If there are too many jets in the central region, such that their resolution becomes difficult-especially if $l_{\mathrm{w}}$ is substantially bigger than 60 - one may reduce the angular range within the central detector or simply watch out for an impressive "jet-fireball topology" in the central region! 
In the near-forward region $(\eta>2.5)$ resolution of individual jets will often be impossible. Except for the case of a low $E_{\mathrm{w}}^{\text {crit }}$ and for partons with typical threshold kinematics (cf. the discussion in sect. 4), one typically expects around $30-40 \%$ of the jets in either the forward or backward region, depending on the shift (5.7) (cf. fig. 4, for $E_{\mathrm{w}}^{\text {crit }}=10 \mathrm{TeV}$ ). For such events one may use triggers requiring a high total hadron multiplicity in the forward and/or backward regions. The strong interaction background can be reduced considerably by restricting the multiplicity trigger region to an annulus with $5^{\circ}<\vartheta<10^{\circ}$ and $170^{\circ}<\vartheta<175^{\circ}$, such that all hadrons produced via strong interactions with $|\eta|>3.25$ are cut out. For GFI events, there remains substantial hadronic activity in this angular region. For $E_{\mathrm{w}}^{\text {crit }}=10 \mathrm{TeV}$ and $l_{\mathrm{w}}=60$ one expects around 10 jets (cf. fig. 5), corresponding to about 100 charged hadrons. (We count 10 charged hadrons per jet, corresponding to an average of 21 charged hadrons for the hadronic decays of the $\mathrm{W}$ bosons.) Some of the events will exhibit a typical asymmetry as to the "deposited" multiplicity in the forward and backward regions, resulting from the shift (5.7).

At this point let us also emphasize that a much more detailed insight into the structure of GFI events can be obtained if a determination of the parton kinematics $\left(x_{\mathrm{a}}, x_{\mathrm{b}}\right)$ is possible for each event. This will require a good calorimetry in the forward and backward regions, such that total energy and momentum of the event can be determined. At the LHC (SSC) the calorimetry would have to cover pseudo rapidities of up to $|\eta| \approx 4.7$ (5.4).

\section{Signal and background}

In conclusion, geometrical flavour interactions are characterized by events with a total transverse energy in the $\mathrm{TeV}$ range, with a high number of isolated leptons, high jet activity in the central region, high hadronic multiplicities and missing transverse energy of several hundred $\mathrm{GeV}$. The main background comes from the tails of strong interaction processes where leptons are produced from semi-leptonic decays of heavy quarks. Near the threshold of detectability, the effective cross section (cf. eq. (3.3)) is only of order $\sum_{i j} \sigma_{0} R_{i j} \approx 10^{-2} \mathrm{pb}$. At the LHC, the QCD cross section for 6 widely separated jets $\left(\theta_{\mathrm{jj}}>50^{\circ}\right)$ with $p_{\mathrm{t}}>20 \mathrm{GeV}$ exceeds this value by seven (!) orders of magnitude [16].

In order to reduce the huge QCD background we propose to use criteria based on total transverse energy deposition and on the expected characteristics of GFI events in the central and forward/backward regions, respectively.

One cut refers to the total transverse energy and the total missing transverse momentum

$$
\begin{aligned}
& E_{\mathrm{t}}^{\mathrm{tot}}>700 \mathrm{GeV} \\
& p_{\mathrm{t}}^{\text {tot }}>50 \mathrm{GeV} .
\end{aligned}
$$


Another one is to select events containing more than two clearly isolated charged leptons with $p_{\mathrm{t}}>25 \mathrm{GeV}$ in the central region. The isolation criteria should be optimized such that the strong interaction background (mainly from decaying $t, b, c$ quarks) is effectively reduced. (One has to keep in mind, however, the high central jet activity of GFI events). In addition, one may focus on a high hadronic multiplicity in the angular range $5^{\circ}<\vartheta<10^{\circ}$ and/or $170^{\circ}<\vartheta<175^{\circ}$. This may be combined with multiplicity information from the central detector. For example, one may require at least 300 charged hadrons in the range $5^{\circ}<\vartheta<175^{\circ}$.

These selection criteria reduce the background from strong interaction events considerably without affecting much the GFI event rate. Subsequently, the detailed leptonic and hadronic structure of the events has to be analyzed in the central region. This strategy hopefully results in a clean distinction from QCD events. An appropriate Monte Carlo analysis would be useful to demonstrate this. We believe that GFI events stand a very good chance to be seen, even if the rate is near the threshold of detectability.

\section{A factory for W's, scalars and top?}

In the preceding section we have concentrated on the case where $\sigma_{\mathrm{w}}$ is relatively small $\left(\sigma_{\mathrm{w}}^{0} \approx 1 \mathrm{nb}\right)$ and the threshold relatively high such that the total number of events is modest and has to be cleanly separated from the QCD background. It seems worthwhile to consider also an optimistic scenario of a low threshold, $E_{\mathrm{w}}^{\text {crit }}=3.5 \mathrm{TeV}$, say, along with a constant cross section at the upper end of the estimate (3.1), $\sigma_{\mathrm{w}}^{0}=10 \mu \mathrm{b}$. In this case the LHC would produce (for $\mathscr{L}=10^{33}$ $\mathrm{cm}^{-2} \mathrm{~s}^{-1}$ ) several thousand GFI events per second (cf. fig. 1)! Every event would presumably include more than ten weak gauge bosons and one or several Higgs bosons. In this case, the LHC turns into a "W, $\mathrm{Z}$ and Higgs factory"! A fruitful exploration of detailed properties of these particles would become possible. The number of top quarks per event depends on the relative probability to produce primordial fermions compared to gauge bosons. Since the coupling and expected mass of the top quark are in a similar range as for the gauge bosons it is conceivable that all weak particle species are produced with similar abundances. Even if the relative rate for the primordial production of a fermion anti-fermion pair of a specific flavour is suppressed by a factor $\alpha_{w}$ compared to gauge boson production, our optimistic scenario would still imply that the LHC and SSC also turn into top factories, with perhaps around one $\mathfrak{t} \bar{t}$ pair per event!

\section{Violation of baryon and lepton number}

If $B+L$ violation becomes strong in the $\mathrm{TeV}$ range, the topology of the $B+L$ violating events will, presumably, resemble roughly the one corresponding to GFI events described above (for $f_{w}$ not much bigger than one). It will be rather central 
(perhaps almost isotropic) close to the threshold energy and increasingly forward oriented as the parton energy increases beyond the threshold. Strong $B+L$ violating cross sections near the unitarity bound would imply strong $B+L$ conserving cross sections, too. This follows immediately from summing over intermediate $B+L$ violating channels for multi-particle amplitudes. Thus, one expects the ratio of $B+L$ violating over $B+L$ conserving events to be at most around one half. If $B+L$ violating and conserving interactions would, indeed, have comparable strengths, it would indicate a common underlying mechanism (i.e. similar classical configurations in an appropriate functional integral representation of the $S$ matrix [12]) and therefore, probably similar kinematics. As a consequence, a distinctly different topology of $B+L$ violating and conserving events seems only reasonable if the $B+L$ violating event rate is small as compared to the $B+L$ conserving one. Even in this case, however, a similar topology seems more likely, since the main characteristic features of GFI events only follow from considerations on the total available kinetic energy (for $\sqrt{\hat{s}}$ near threshold), slow multiplicity increase and limited transverse momentum (for $\sqrt{\hat{s}}>E_{w}^{\text {crit }}$ ). If $B+L$ violating cross sections really become strong at some threshold, it seems more plausible that they remain almost constant at energies above the threshold. (An asymptotic decrease $\sigma_{\Delta(B+L)} \sim S^{-1}$ would imply the unlikely scenario that strong $B+L$ violation is only a transient phenomenon in a narrow energy window). Partial wave unitarity then implies that the events must become more forward oriented as $\sqrt{s}$ increases. In any case, at threshold machines like the LHC or SSC, we expect many very central GFI events and a distinction of $B+L$ violating events by topology or kinematical considerations seems almost impossible.

We conclude that $B+L$ conserving GFI events are the dominant background for a possible detection of electroweak $B+L$ violation at high energies. Since the background has a similar event topology, a search for $B+L$ violation should directly concentrate on quantum numbers. We propose to exploit that the $B+L$ violating interaction

$$
\mathrm{q}+\mathrm{q} \Rightarrow 7 \overline{\mathrm{q}}+3 \bar{l}+\mathrm{X}
$$

produces on average more positrons and $\mu^{+}$than electrons and $\mu^{-}$. This allows us to measure the average lepton charge asymmetries

$$
\frac{\left\langle N_{\mathrm{e}^{+}\left(\mu^{+}\right)}-N_{\mathrm{e}^{-\left(\mu^{-}\right)}}\right\rangle}{\left\langle N_{\mathrm{e}^{+}\left(\mu^{+}\right)}+N_{\mathrm{e}^{-\left(\mu^{-}\right)}}\right\rangle}
$$

for isolated electrons and muons (with $p_{\mathrm{t}}>20 \mathrm{GeV}$ ) in the central region. The 
asymmetry vanishes for lepton number conserving GFI events up to small effects due to the like-sign charge of the incoming protons.

As to the usefulness of the average charge asymmetries (11.2), two comments are in order.

(i) Since the charges for electrons and muons are almost uncorrelated for a given event (see sect. 6), we expect rather large fluctuations of $N_{\mathrm{e}^{+}\left(\mu^{+}\right)}-N_{\mathrm{e}^{-}\left(\mu^{-}\right)}$on an event by event basis. For $B+L$ conserving interactions the average value of the asymmetry should only vanish for a large number of events, with a statistical error determined by the average size of the fluctuations and the number of events.

(ii) Lepton number conservation implies $\left\langle N_{\mathrm{e}^{+}\left(\mu^{+}\right)}-N_{\mathrm{e}^{-}\left(\mu^{-}\right)}\right\rangle \approx 0$ only if the experimental cuts (like e.g. angular cuts, isolation cuts or $p_{\mathrm{t}}$ cuts) are completely symmetric with respect to the lepton charge distribution. This is far from trivial, since an asymmetry in the kinematic distributions of $\mathrm{W}^{+}$and $\mathrm{W}^{-}$, for example, may reflect itself in an induced effective charge asymmetry of the lepton cuts. To minimize such possible effects it seems advantageous to concentrate on isolated leptons (with $p_{\mathrm{t}}>30 \mathrm{GeV}$ ) in a central rapidity region.

We conclude that at best a relatively strong $B+L$ violation (as compared to the GFI background) could be detected by a measurement of leptonic charge asymmetries. Given the multiparticle environment, this will be a delicate task. As an alternative, one may search for special properties of the primordial fermions to filter out the $B+L$ violating events. This has, however, to compete always with the "tails" of geometrical flavour production.

\section{Conclusions}

We have discussed strategies to detect possible signals of "geometrical" flavour production in the $\mathrm{TeV}$ regime. The corresponding events are supposed to occur for parton c.m. energies exceeding a certain (critical) threshold energy $E_{\mathrm{w}}^{\text {crit }} \approx 2-20$ $\mathrm{TeV}$ and are characterized, first of all, by the production of many weakly interacting "particles" ( $=$ gauge bosons, quarks, leptons, Higgs; $n_{\mathrm{w}}=\mathrm{O}\left(\alpha_{\mathrm{w}}^{-1}\right) \approx 30$ ) with a relatively large, almost energy-independent parton cross section $\sigma_{\mathrm{w}}^{0} \approx 0.1 \mathrm{nb}-10$ $\mu \mathrm{b}$. The transverse momenta of the produced particles reflect the "weak" size of the scattering quarks $\left(\alpha m_{\mathrm{w}}^{-1}\right)$ and are of the order of 30-50 GeV. We concentrate on the light fermions of mass smaller than the transverse momentum, which may be produced both promptly and as decay products of $\mathrm{W}, \mathrm{Z}$ bosons etc. The associated jets and charged leptons are directly accessible experimentally.

We find the following characteristic features of "geometrical flavour interaction" (GFI) events (for $n_{\mathrm{w}}=30$ ):

(i) For parton kinematics near the threshold $\left(\sqrt{\hat{s}} \approx E_{\mathrm{w}}^{\text {crit }}\right)$, the events look rather central, i.e. jets and charged leptons are distributed over the whole angular range. Only partons with energies much above $E_{\mathrm{w}}^{\text {crit }}$ produce more forward oriented events. For "threshold machines" with $\sqrt{s}=16-40 \mathrm{TeV}$ most of the activity will be 
in the central detector $\left(10^{\circ}<\vartheta<170^{\circ}\right)$. Even at $\sqrt{s}=200 \mathrm{TeV}$ one may expect on the average about one half of the produced particles in this central region.

(ii) The average transverse momentum per (light) particle is estimated as $\bar{p}_{\mathrm{t}}^{1} \approx 35$ $\mathrm{GeV}$ and the total transverse energy is large, $\left\langle E_{\mathrm{t}}^{\text {tot }}\right\rangle \approx 1.6-2.2 \mathrm{TeV}$.

(iii) We expect on average at least 3.5 (more typically 5) "isolated" electrons or muons per event.

(iv) A similar number of neutrinos is responsible for an average missing transverse momentum $\left(p_{t}^{\text {tot }}\right) \approx 100 \mathrm{GeV}$.

(v) More than 20 jets/event should be seen in the central detectors.

(vi) In addition, we expect many events with a high charged hadron multiplicity $\left(n_{\mathrm{h}}>100\right)$ in the forward and/or backward detectors $(|\eta|>2.5)$.

We believe that GFI events stand a good chance to be seen at future colliders if the threshold energy $E_{w}^{\text {crit }}$ is not too high (e.g. below 11 (28) $\mathrm{TeV}$ for the LHC (SSC), in case of a parton cross section of $\sigma_{\mathrm{w}}^{0} \approx 1 \mathrm{nb}$ ). For larger cross sections and lower $E_{\mathrm{w}}^{\text {crit }}$ these machines may turn into W, Higgs, and top factories with possibly as many as $10^{3} \mathrm{GFI}$ events per second!

We thank W. Bartel, G. Schuler and J. Vermaseren for useful discussions.

\section{References}

[1] A. Ringwald and C. Wetterich, Nucl. Phys. B353 (1991) 303

[2] V. Fadin, E. Kuraev and L. Lipatov, Phys. Lett. B60 (1975) 50;

E. Kuraev, L. Lipatov and V. Fadin, Sov. Phys. JETP 44 (1976) 443; 45 (1977) 199;

Y. Balitsky, L. Lipatov, Sov. Journ. Nucl. Phys. 28 (1978) 822; JETP Lett. 30 (1979) 355;

J. Bartels, Nucl. Phys. B151 (1979) 293; B175 (1980) 365;

Y. Balitsky, L. Lipatov and V. Fadin, in Materials of the 14th Winter School of Leningrad Institute of Nuclear Research (1979), p. 109, (in Russian);

L. Lipatov, Sov. Phys. JETP 63 (1986) 904

[3] H. Aoyama and H. Goldberg, Phys. Lett. B188 (1987) 506

[4] A. Ringwald, Nucl. Phys. B330 (1990) 1

[5] O. Espinosa, Nucl. Phys. B343 (1990) 310

[6] L. McLerran, A. Vainshtein and M. Voloshin, Phys. Rev. D42 (1990) 171

[7] N. Manton, Phys. Rev. D28 (1983) 2019;

F. Klinkhamer and N. Manton, Phys. Rev. D30 (1984) 2212

[8] A. Belavin, A. Polyakov, A. Schwartz and Y. Tyupkin, Phys. Lett. B59 (1975) 85

[9] G. 't Hooft, Phys. Rev. D14 (1976) 3432; D18 (1978) 2199

[10] I. Affleck, Nucl. Phys. B191 (1981) 445

[11] V.I. Zakharov, TPI-MINN-90/7-T (1990)

[12] S. Khlebnikov, V. Rubakov and P. Tinyakov, Nucl. Phys. B350 (1991) 441

[13] M. Porrati, Nucl. Phys. B347 (1990) 371

[14] J.M. Cornwall, talk at workshop Electroweak baryon number violation up to $200 \mathrm{TeV}$ (Erice 1990)

[15] J. Whitmore, Phys. Rep. C10 (1974) 273

[16] Z. Kunszt, in Proc. Physics at future accelerators, La Thuile, 1987, CERN 87-07, Vol 1 